\title{
Simplified Tai Chi 6-Form Apparatus for Balance in Elderly People with Alzheimer's Disease
}

\author{
Yin-Chou Lin ${ }^{1}$ (1) Wen-Chuin Hsu ${ }^{2,3}$. Chien-Hung Chen ${ }^{4}$ Chai-Wei Wang ${ }^{1} \cdot$ Katie Pei-Hsuan $\mathrm{Wu}^{4,5}$. \\ Alice M. K. Wong ${ }^{1,5}$
}

Received: 30 January 2018 / Accepted: 9 October 2018 / Published online: 25 October 2018

(c) The Author(s) 2018

\begin{abstract}
Background People with Alzheimer's disease (AD) can have deteriorated walking and impaired postural-balance control, which increases the risk of falls. Tai chi (TC) can improve fitness and balance in elderly people. To facilitate TC learning, we developed the Simplified Tai Chi 6-Form Apparatus (STC6FA).

Methods This case-controlled study enrolled 26 elderly people with mild-to-moderate AD. Over 8 weeks, the TC group (TCGr) of 11 elderly people with AD completed a training course for the STC6FA. Another 10 older adults with matched age, height, and severity of dementia were assigned to the control group (CGr). Balance function was measured using a SMART Balance Master (NeuroCom International Inc., OR, USA). The parameters were evaluated three times during the training course, at weeks 0,4 , and 8 .

Results The balance of both the TCGr and CGr improved significantly. A rhythmic forward-backward shift test revealed more improvement in the TCGr than in the CGr.

Conclusion STC6FA training can improve balance and may reduce backward falls in elderly people with mild-to-moderate $\mathrm{AD}$.
\end{abstract}

Keywords Dementia $\cdot$ Equilibrium $\cdot$ Falls $\cdot$ Frail elderly people $\cdot$ Tai chi

JEL Classification I100 Health · General

\section{Introduction}

Taiwan's population is aging, as are those of many other countries. The percentage of Taiwanese people at and over 65 years old was $13.86 \%$ in June 2017 [1]. Approximately

Alice M. K. Wong

alicewong.mk@gmail.com

1 Department of Physical Medicine and Rehabilitation, Chang Gung Memorial Hospital, 5, Fu-Hsin St., Kwei-Shan, Tao-Yuan 333, Taiwan, ROC

2 Department of Neurology and Dementia Center, Chang Gung Memorial Hospital, Tao-Yuan, Taiwan, ROC

3 Healthy Aging Research Center, Chang Gung University, Tao-Yuan, Taiwan, ROC

4 Department of Physical Medicine and Rehabilitation, Chang Gung Memorial Hospital, Linkou, Taiwan, ROC

5 School of Medicine, Chang Gung University, Tao-Yuan, Taiwan, ROC
$35-40 \%$ of people aged 65 years and older experience fall injuries every year. The risk of being seriously injured in a fall increases with age. Injuries can occur in $5-15 \%$ of fall incidents in elderly people [2]. The risk factors for fallrelated injuries include multiple medical illnesses, cognitive impairments, neuromuscular impairments, balance and gait disorders, depression, functional decline, multiple drug use, and environmental hazards [3, 4].

Changes in gait performance have been associated with increased fall risk in elderly people, especially during dualtask gait changes (e.g., when a person stops walking while speaking) [5]. Impairment of gait is found to be one of the most consistent predictors of falls and a prevalent feature in elderly people with cognitive impairment.

People with Alzheimer's disease (AD) have been observed to have deteriorated walking and impaired postural-balance control early in the course of the disease [6]. Difficulty with postural control has been linked to a decline in cognitive and sensorimotor function related to the disease 
because gait control requires cognition, particularly the executive functions required for planning and working memory while performing a more complex gait task [7]. In patients with moderate-to-severe $\mathrm{AD}$, prominent disequilibrium, short steps, and shuffling are common disorders, including start-and-turn hesitation during walking [8]. These motor signs have been associated with an increased risk of falls. People with AD were found to have a three-fold increased risk of falls and two-fold increased risk of fractures over cognitively intact elderly people [9].

Physical exercise has been proven to benefit elderly people in terms of mobility, physical function, and fall prevention [10]. Exercise is also recommended for managing dementia. Evidence shows that regular physical activity is positively associated with minimizing cognitive decline in elderly people [11]. Regular exercise has been associated with a relatively low rate of decline in the activities of daily living in nursing home residents with AD [12]. However, elderly people may experience great difficulty when attempting to exercise regularly in their communities due to low motivation and a lack of social support. In Taiwan, commercial fitness centers usually reject elderly clients. Thus, elderly people often lack safe environments with exercise devices that would enable them to maintain a regular exercise habit.

Tai chi (TC), also known as tai chi chuan, is a Chinese conditioning exercise suitable for elderly people and even for patients with chronic disease. Basic TC exercise is composed of a series of graceful motions linked together as a flowing sequence in which the body is continuously shifting from one foot to the other with a low center of gravity. TC is also a mind-body exercise. Practicing TC can train people to perform dual-task activities because the practitioner's eye focuses on the leading arm and the mind focuses on the breath and movement sequences. Our previous studies have proven that TC can improve fitness, balance, and hand-eye coordination in elderly people [13-15].

Lam et al. reported that a simplified form of TC improved the cognitive function of elderly participants as well as their coordinated-cognitive integration of attention, voluntary motor actions, and postural control [16]. TC practice demands constant concentration, coordination, and integration of the motor and language systems. A recent meta-analysis found that TC has a positive effect on people with mild cognitive impairment who are at risk of cognitive decline [17]. Another meta-analysis showed that TC has beneficial effects on depression, anxiety, general stress management, and exercise self-efficacy [18]. Therefore, TC seems to be a promising exercise intervention for elderly people with AD. However, attention and executive impairment are frequently found to be associated with physiological aging and dementia [19]. Therefore, it is not easy for people with AD to learn TC and practice by themselves. To counter this, we developed the Simplified Tai Chi 6-Form Apparatus (STC6FA) to provide a simple method of learning TC. We performed clinical assessments on healthy elderly people and stroke patients who were using the STC6FA. We found that the STC6FA is easy to use for healthy elderly people and even for stroke patients; in addition, the apparatus is safe and yields satisfactory results [20]. In this study, we examined the effects of using the STC6FA on the balance of elderly people with mild-to-moderate AD.

\section{Methods}

\subsection{Participants}

The protocol used in this study was reviewed and approved by the Institutional Review Board of Chang Gung Memorial Hospital, Taiwan (IRB No. 103-5846C). The clinical trial registry number was NCT02978768. Participants with $\mathrm{AD}$ exhibiting mild-to-moderate cognitive impairment were recruited from the Dementia Center of Chang Gung Memorial Hospital from May 2012 to December 2013.

Identification and diagnosis of $\mathrm{AD}$ was performed at our memory clinic according to the standard protocol of the National Institute of Neurological and Communicative Disorders and Stroke and the AD and Related Disorders Association [21]. The Mini-Mental State Examination (MMSE) and Clinical Dementia Rating (CDR) scale are critical parts of the diagnostic protocol [22]. The inclusion criteria were patients with mild-to-moderate $\mathrm{AD}(\mathrm{CDR} \leq 2)$, who could follow clearly administered orders. Those with heart disease, lung disease, a bone problem (e.g., history of fracture, knee or hip joint replacement, or amputation), a neurological disorder (e.g., cerebrovascular disease, parkinsonism), or contraindication for moderate-intensity exercise training were excluded.

\subsection{Simplified Tai Chi 6-Form Apparatus}

These six forms of TC were jointly developed by a TC master and a physician who practiced TC (Patent of Taiwan I333862, I333863, I333864, I330537, I335827).

(a) Start up form: preparation to warm up the mind and body for exercise (Fig. 1).

(b) Cloud hand form: shift body weight from side to side with upper and lower limbs moving in the same direction (Fig. 2).

(c) Opening and closing the hands: chest expansion using the arms and hands; expand the chest from midline to sideline (Fig. 3).

(d) TC palm: shift body weight from front to back by hand manipulation with a ball (Fig. 4). 

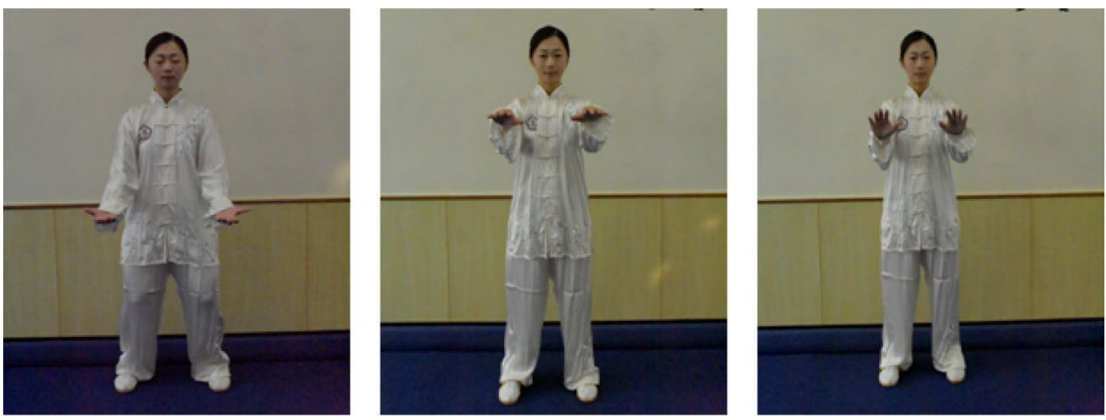

(a)

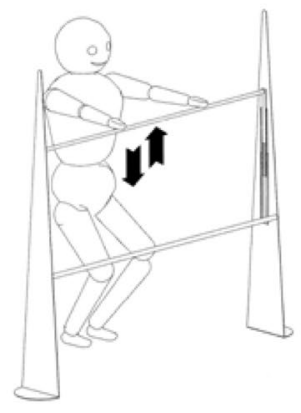

(b)

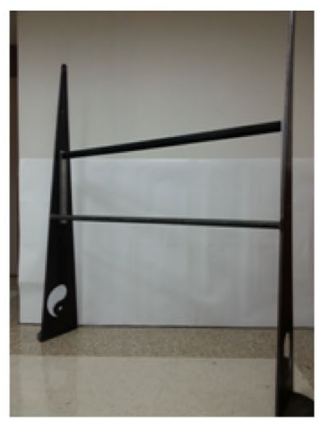

(c)
Fig. 1 Starting Posture: commencing form. a Decomposition diagrams. b Design diagram for the Starting Posture. c Position your legs in the semi-squat posture. Stretch your arms out in front of you at an equal height to your shoulders. Move your legs, arms, and trunk

(e) Oblique flying form: move body up and down; move upper and lower limbs in a cross direction with a sideto-side movement (Fig. 5).

(f) Rooster standing on one leg: stand on one leg with a raised arm for balance (Fig. 6).

\subsection{Intervention}

A total of 26 elderly persons with $\mathrm{AD}$ and mild-to-moderate dementia were enrolled. All of the participants were community dwellers who were arranged into a TC group (TCGr) and a control group (CGr) according to the timing of their recruitment (the first 13 patients were the TCGr and the remainder were the CGr). Because of changes in their physical condition, five participants were unable to finish the study (one participant fell at home and fractured a bone, one experienced a vertigo attack, and three others could not maintain the weekly schedule). In the TCGr, nine participants were mild $\mathrm{AD}$ and two were moderate; in the $\mathrm{CGr}$, all participants were mild AD. The 11 elderly participants with AD completed the training course for the STC6FA for 8 weeks, two times per week, and 45 min per session. The 6-form TC was originally designed by Professor Lan, who was a silver medalist in the Asian Games. Each mild-to-moderate $\mathrm{AD}$ patient was guided by a designated instructor to simultaneously while maintaining natural breathing. The instrument depicted in the picture is designed to increase the leg stability, and enhance the strength of your waist muscles. d Product for the Starting Posture

ensure the movements were performed accurately. Another 10 elderly persons with $\mathrm{AD}$ were selected for the $\mathrm{CGr}$ and led in stretching exercises instead of the STC6FA for the same 8-week schedule. The STC6FA was designed to create an athletic apparatus that is effective and simple for elderly people to use. Ideally, TC exercises, including major muscle group exercises but excluding jumping exercises, can be selected to increase lung capacity, engage in joint range of motion, and achieve muscle flexibility. The aforementioned six gestures (starting TC, cloud hand, opening and closing the hand, TC palm, oblique flying, and rooster standing on one leg) were selected to design the STC6FA. The STC6FA was modified to try and avoid the confounding effect of limited memory in AD patients and ensure execution accuracy. The newly developed STC6FA has passed an intellectual property office evaluation in Taiwan. The stretching exercises of the CGr include neck stretch, triceps stretch, shoulder stretch, shoulder circles, upper back extension, chest stretch, hip flexion, knee flexion, ankle plantar flexion, and step exercises (Fig. 7). All participants were guided by a qualified exercise instructor at each session. Compared with traditional TC training, the STC6FA makes learning TC movements easier, and thus provides elderly people with a chance to learn that they may not otherwise have. However, the STC6FA has only limited styles compared with 

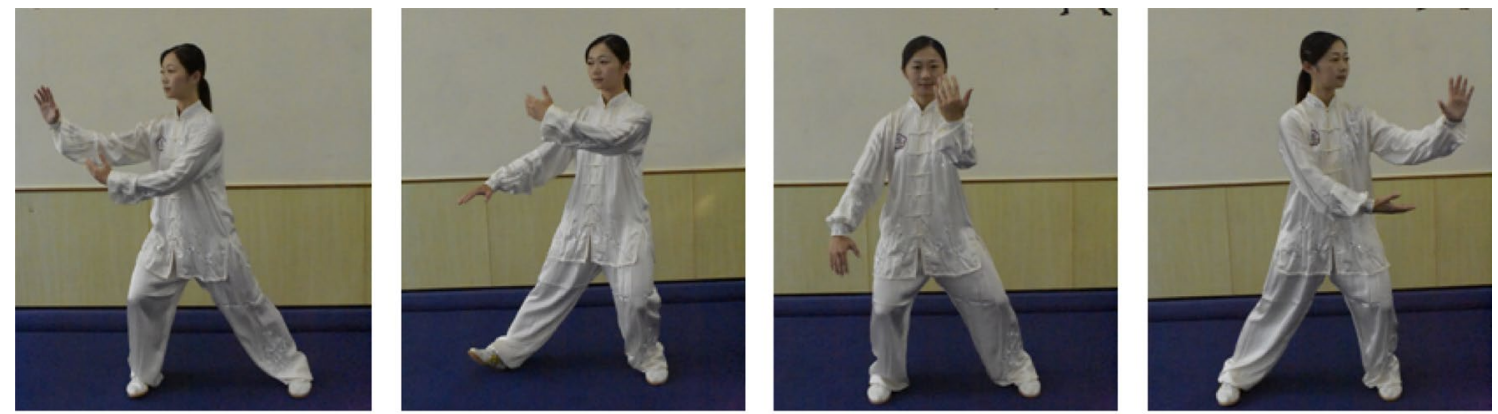

(a)

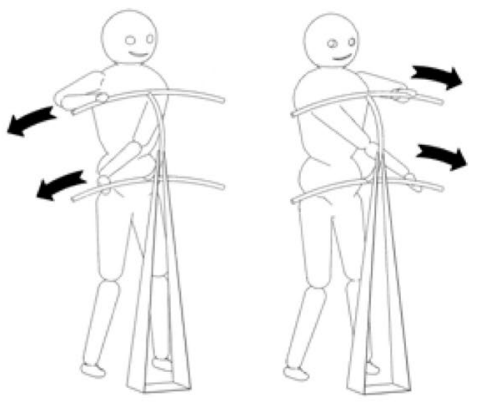

(b)

Fig. 2 Cloud Hand: wave hands like clouds. a Decomposition diagrams. b Design diagram for the Cloud Hand. The instrument depicted herein comprises two fixed and parallel curved bars. Place your hands on the beams and slide along them. Stand with your feet shoulder width apart and relax your shoulders. Raise one hand to the middle of your chest and place the other hand $3 \mathrm{~cm}$ below your belly

traditional TC, and it is not suitable for people who are too tall (above 95th percentile of male body height) or too short (below 5th percentile of female body height) as they are unable to fit the frame of the STC6FA.

\subsection{Outcome Measurements}

The changes in participants' mental state and dementia scores were measured respectively by the MMSE and Behavioral pathology in Alzheimer's disease (BEHAVE$\mathrm{AD}$ ) rating scale; participants' depression scores were measured using the Cornell Scale for Depression in Dementia (CSDD); their balance was measured using a SMART Balance Master (NeuroCom International Inc., OR, USA). The measurements were taken three times during the training course, at weeks 0,4 , and 8 . A flow diagram is shown in Fig. 8.

\subsubsection{Balance Testing}

A SMART Balance Master was employed to measure postural stability under defined conditions. The device consisted of two force plates, a visual enclosure, an overhead bar and safety harness, and computer hardware and software. The

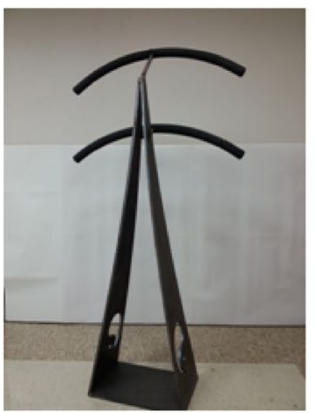

(c) button. Move your hands and torso left and right following your legs while maintaining natural breathing. The instrument is designed to increase the stability of your legs, assist in the sideward stretching of your torso and improve the strength of your waist muscles. $\mathbf{c}$ Product for the Cloud Hand

force plates were $0.229 \times 0.457 \mathrm{~m}^{2}$ and rested on individual stands. The two force plates were supported by four force transducers (strain gauges) that were mounted symmetrically on a supporting center plate. The visual enclosure, which was composed of a wall covered with grey aluminum sheeting and a small blue-dot pattern, enclosed the participant on three sides. A computer monitor was located approximately $0.6 \mathrm{~m}$ in front of the participant at eye level. The machine had an overhead bar and harness to ensure the participant's safety while the measurements were performed. A computer with a intel 486-series CPU ran the SMART Balance Master software. This entire system was automatically calibrated by an internal system. The force plate data were sampled at $100 \mathrm{~Hz}$.

2.4.1.1 Static Postural Stability Test A sensory organization test (SOT) evaluated the participants for postural stability by using the following sequence: (1) eyes open and fixed on the support (EO); (2) eyes closed and fixed on the support (EC); (3) sway-referenced vision and fixed on the support (SV); (4) eyes open and sway-referenced support (SS); (5) eyes closed and sway-referenced support (ECSS); and (6) sway-referenced vision and support (SVSS). A complete protocol included two single trials of 

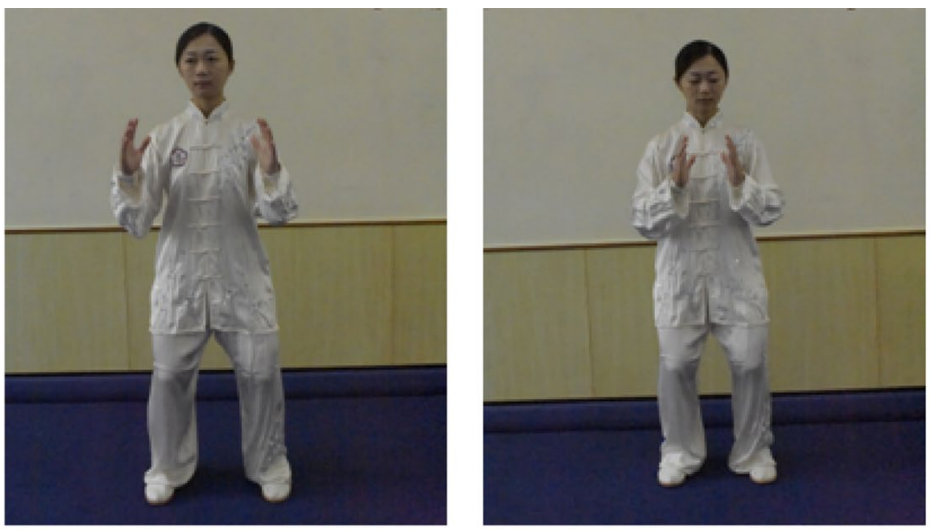

(a)

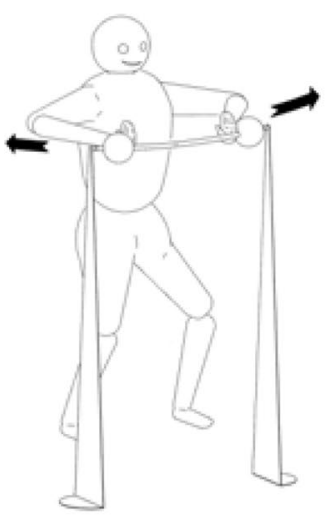

(b)

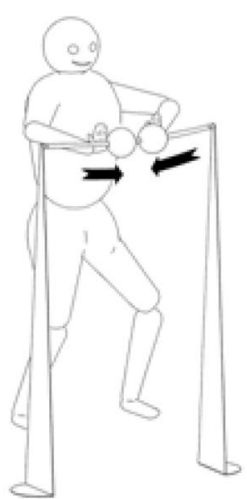

Fig. 3 Hand Opening and Closing: open and close hands in a semisquatting posture. a Decomposition diagrams. b Design diagram for the Hand Opening and Closing. The instrument depicted herein consists of two spheres attached to a curved bar. Grab the spheres with your hands. Stand with your feet shoulder width apart. Adjust the range of activity according to the distance you can create between your feet in a semi-squatting posture.. Relax your shoulders, and clasp your hands, and position them in front of your nose. Stand firm

EO and EC conditions and three consecutive trials for the remaining conditions. Each trial lasted $20 \mathrm{~s}$. Before every trial, the participants were informed of the conditions, such as whether the test would be EO or EC and whether the visual surroundings or the floor would move.

2.4.1.2 Dynamic Postural Stability Test The rhythmic weight-shift (RWS) test was performed by asking the test participants to shift their weight laterally (from left to right) or anteroposteriorly (from front to back) on the platform. The participants were instructed to follow a cue (a yellow sun) on a screen that moved between two end points (displayed as red lines). The cue moved at three speeds (slow, medium, and fast) corresponding to each directional condition. Verbal instructions and practice time were given to each participant. Transition times for the cue speeds were as follows: the slow cue speed was

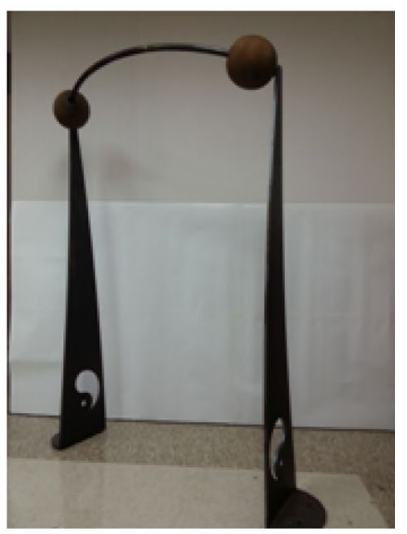

(c)

and open and close your clasp (standing palm), and the opening distance should not exceed that of the inner shoulder. Inhale air into the lower Dantian as you open your clasp (feel your abdomen expand), and exhale as you close your clasp. This instrument increases the stability of your legs, trains your shoulder, and enhances the strength of your upper arm muscles. Repeated practice can improve pulmonary function. c Product for the Hand Opening and Closing

$3 \mathrm{~s}$, medium was $2 \mathrm{~s}$, and fast was $1 \mathrm{~s}$. All trials were assessed and scored.

\subsection{Data Analysis}

In the balance testing, the SMART Balance Master offered the following outcome measures for postural stability: (1) the equilibrium score was a measure of maximal stability, represented by a percentage on the SOT. A relatively high percentage represented reasonably stable postural balance (100\% indicated little swing, and $0 \%$ indicated a loss of balance). (2) A measure of the center of pressure was represented by a velocity change in degrees per second on the RWS test: the higher the velocity was, the less stable the control was. 

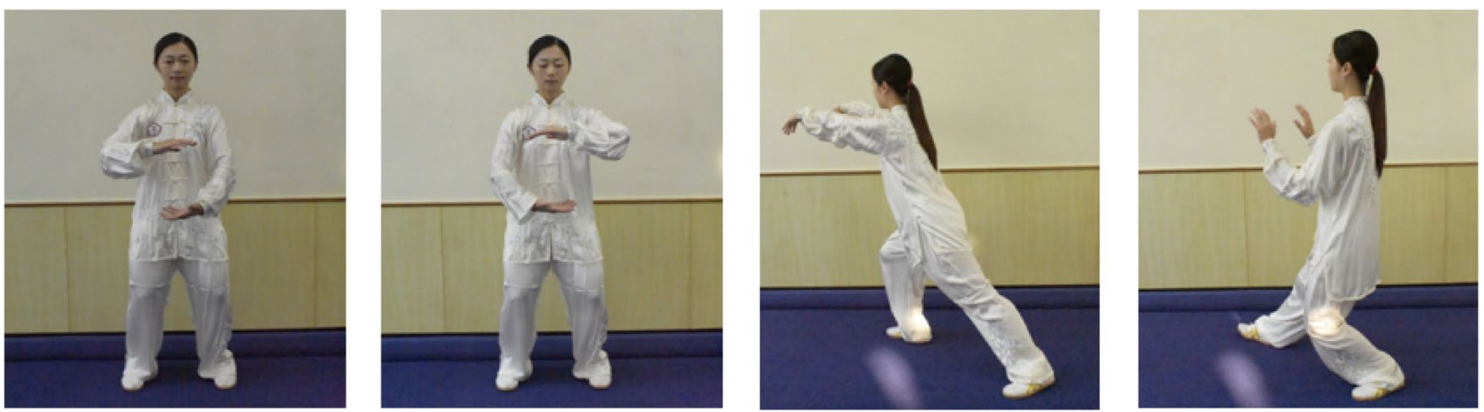

(a)
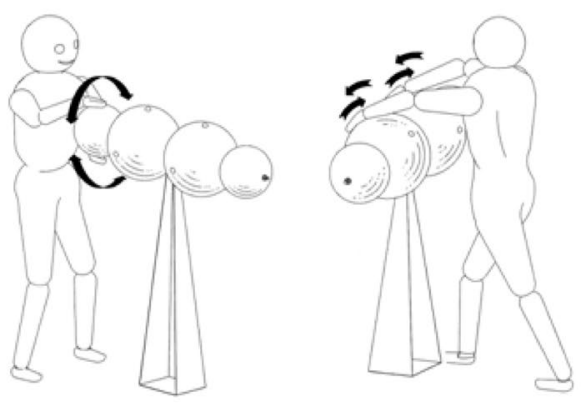

(b)

Fig. 4 Tai Chi Palm: Tai Chi round form. a Decomposition diagrams. b Design diagram for the Tai Chi Palm. This instrument features four spheres attached to one cross bar. For the movement focusing on the small sphere, first stand with your feet shoulder width apart. Next, hold the lower side of one small sphere with your palms in a similar manner to holding a basketball. Stably and slowly pull your palms away from the sphere, maintaining your posture with your eyes closed. For another movement, stand with your feet shoulder width apart again. Hold the small sphere on its lower and upper sides with your palms and rotate it lightly. Expand the rotation angle $\left(180^{\circ}-\right.$ $270^{\circ}$ ) as you loosen your hold. When said angle reaches $270^{\circ}$, press

\subsection{Statistical Analysis}

Nonparametric statistics were used to compare the differences in age, body weight, and body height between the two groups. A repeated-measures analysis of variance was used to compare the two groups in balance testing, vital capacity, grip force, and body composition as well as MMSE, BEHAVE-AD, and CSDD scores. Data were expressed as means with standard deviations. Statistical significance was set at $P<0.05$. SPSS version 22.0 (IBM Corporation, Armonk, NY, USA) was used to process the data.

\section{Results}

Participants in the TCGr had a mean age of $76.7 \pm 11.0$ years $(n=11)$, whereas the CGr participants had a mean age of $76.8 \pm 6.9$ years $(n=10)$; the TCGr had MMSE scores of $17.6 \pm 7.4$ and CDR scores of $0.8 \pm 0.6$, whereas the CGr had MMSE scores of $21.2 \pm 4.2$ and CDR scores of $0.9 \pm 0.2$.

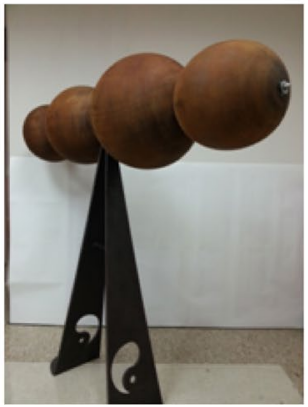

(c) the shoulder linked to the upper hand downward to complete the bending of the arm muscles, leg movements to an oblique step can be performed in conjunction to hold the big spheres with the arm muscle exercise. For the movement that involves both the large and small spheres, grab the spheres to train the muscles of turning your palms with arms up and down. Spin the ball in a top-down direction and exhale when exerting your strength, after which loosen your palm as you push the sphere downward. This instrument increases the stability of your legs and step, trains your shoulder joints, and improves the strength of your upper arm muscles. c Product for the Tai Chi Palm

The TCGr comprised five men and six women, whereas the CGr comprised three men and seven women. As presented in Table 1, no significant differences existed in age, body height, or severity of dementia between the two groups, except that body weight in the TCGr was $63.3 \pm 9.1 \mathrm{~kg}$, whereas it was $53.9 \pm 6.4 \mathrm{~kg}$ in the $\mathrm{CGr}(P<0.05)$.

The TCGr's balance score on the SOT changed from 45.9 \pm 21.7 to $54.7 \pm 15.9(P<0.05)$, whereas the CGr's score changed from $50.5 \pm 10.3$ to $55.9 \pm 15.0(P>0.05)$. Regarding the dynamic tests, the RWS test indicated a significant improvement in movement velocity and direction control in forward-backward movement relative to the CGr. MMSE revealed no significant improvement in both groups (Table 2).

\section{Discussion}

Aging is an issue of concern throughout the world. Dementia has been reported as increasing in incidence with age, with a prevalence of $5-8 \%$ for people aged 65 and over in the 

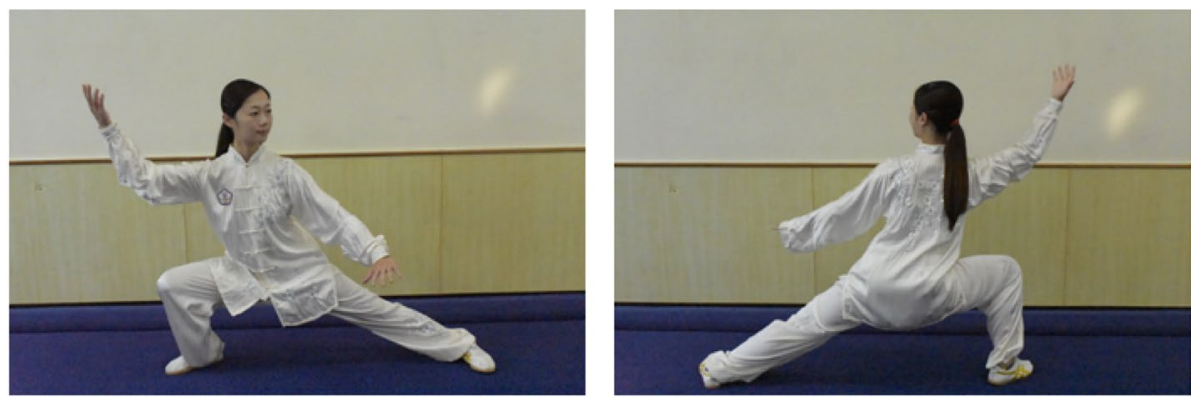

(a)

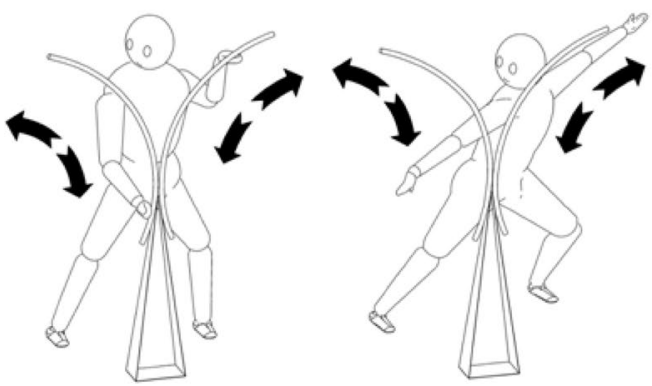

(b)

Fig. 5 Oblique Flying: oblique arm and leg up and down alternately. a Decomposition diagrams. b Design diagram for the Starting Posture. This instrument comprises two upright bars that are curved inward; each is the length of a leg. This instrument increases the stability of your legs by alternating up and down in sideward. Grab the bars and move your palm up and down along it. Maintain your arms in a straight line while stretching your body sideways, raise your arms

United States and of 5.5\% for elderly people in Asia [23]. In elderly people with mild cognitive impairment, fall-related injuries are more likely to contribute to morbidity than in healthy elderly people [24].

Exercise intervention has been shown to be a promising treatment for elderly people with AD. Previous studies have used 12-month randomized control trials to examine the effect of resistance training on balance and muscle tone; these have shown that resistance training improved performance on the Stroop test [25, 26]. Many studies, investigating the effects of exercise on elderly people with dementia have reported an improvement in global cognitive measures, suggesting that an improvement in cognition from regular exercise can be a valid and reliable result [16]. Aerobic exercise has potential benefits on cardiopulmonary fitness, single-task physical performance, dual-task performance, and exercise self-efficacy in community-dwelling patients with mild $\mathrm{AD}$ [27].

TC, a traditional Chinese style of mind-body exercise, was reported to be very effective for postural stability and

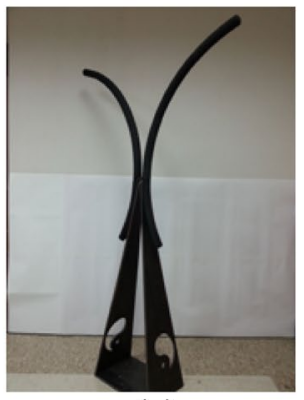

(c) to stretch further as you bend your body. Push your left hand to the upper left and right hand to the lower right to work the trunk and arm muscles. Allow your head to move naturally by following your eyes focusing on your hands, positioning your feet to match the distance between your shoulders with adequate weight shift side by side stably. c Product for the Starting Posture

fall prevention in elderly people over a relatively short period of practice, between 1 and 3 months [28]. TC practice demands constant concentration, coordination, and integration of the motor and language systems. The cognitive benefits may differ from those of stretching exercises, which require a lower level of cognitive demand during practice. Our previous study revealed that the TC group exhibited more improvements of visual attention (visual span). The improvement in visual attention appeared to be consistent with findings that TC demands attention for specific postures and motor sequences [26]. Attention is integral to coordination among major cognitive abilities, and thus attention impairment is frequently found to be associated with physiological aging and dementia. Therefore, researchers should focus their effort on interventions that foster the visual span and that have the potential for cognitive preservation. A study found that TC has positive effects on people with mild cognitive impairment, a group that are at risk of cognitive decline [17]. TC also has beneficial effects on depression, anxiety, general stress management, and exercise 

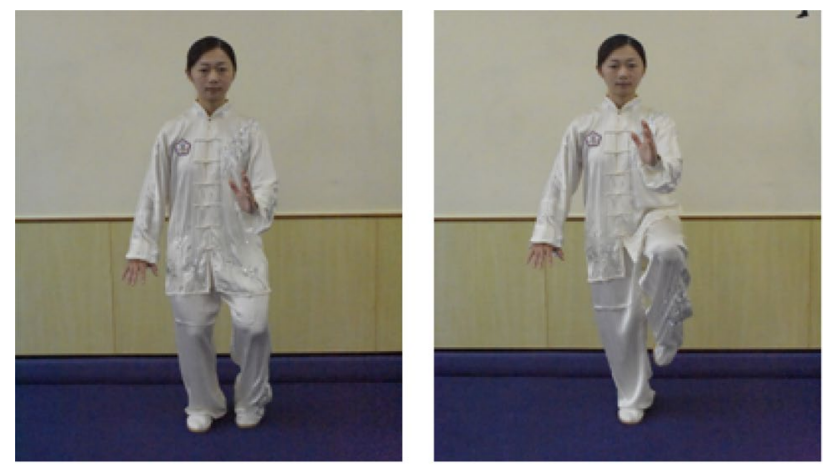

(a)

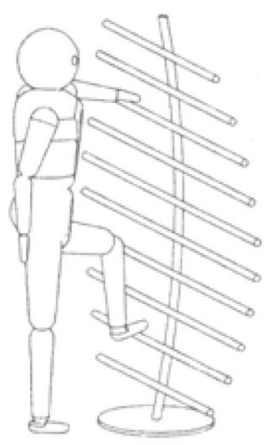

(b)

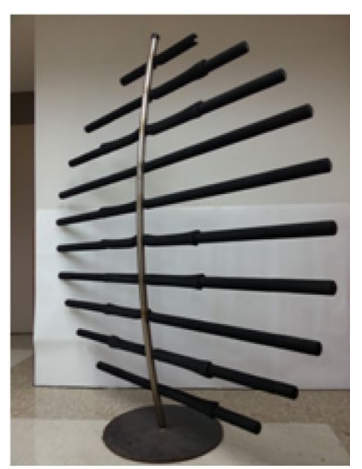

(c)
Fig. 6 Gold Rooster Stance: push down and stand on one leg. a Decomposition diagrams. b Design diagram for the Starting Posture. This instrument features a total of six steps with an interval of $15 \mathrm{~cm}$ between each step. Each step is compatible with the length between the sole and the ankle (when a shoe is worn), and may be adjusted according to an individual's height. Position your feet shoulder width apart, and extend one hand forward at shoulder height until it reaches the location of the upper legs. Maintain the interval between the index finger and thumb at the width of a quail's egg. Slowly raise the knee at the side of your active hand to the height of the upper end of the upper leg, and withdraw the weight of your foot and hand from the instrument. Press the idle hand downward to maintain balance. Use the instrument to increase the stability of your legs, improve the strength of your leg muscles, and train your body balance. c Product for the Starting Posture

self-efficacy [18]. Therefore, TC is likely a suitable exercise option for managing dementia.

TC training can reduce balance impairments and falls in healthy elderly people and in patients with Parkinson disease [29]. However, only a few studies have focused on the effects of TC training on the balance of patients with $\mathrm{AD}$, possibly because patients with $\mathrm{AD}$ are prone to exhibit impaired attention, which impedes learning ability. We developed the STC6FA to help AD patients overcome the obstacles of learning TC. The benefits of the STC6FA are that it is safe and simple to use, and it is suitable for patients or elderly people with mild cognitive impairment. In this study, the SOT balance scores were significantly improved in the TCGr after 8 weeks of training. This finding suggests that TC has positive effects on static postural stability. We also found that the TCGr had better RWS score improvements than did the CGr in forward-backward movement. This finding suggests that the STC6FA can help patients with AD reduce balance impairment (especially backward disequilibrium) both in terms of static and dynamic postural stability.

This study was limited because it was an equivalentcontrol-group trial (i.e., not randomized), and thus it could not provide the same level of evidence that a randomized control trial could. The participants were limited to elderly people with mild-to-moderate AD. Therefore, the results cannot be generalized to all AD patients. Although we provide a free hospital-based day care center program, it is inconvenient for caregivers to send AD patients to our hospital, because AD patients rely on their caregivers for transportation. Therefore, recruitment of AD subjects for this study was difficult, and the number of cases may be insufficient to demonstrate statistical significance. The STC6FA is limited to six forms of TC; hence, the results of this study cannot indicate the effects of traditional TC practice. However, the STC6FA is a novel and simple-touse TC apparatus that is a worthwhile exercise intervention with demonstrable beneficial effects on AD patients. The development and application of such apparatus could safely provide exercise for elderly people with some degree of dementia. The design of the STC6FA might be transferred to suitable sports equipment manufacturers for both indoor and outdoor use. Notably, the participants' body weights did not match between the two groups. Thus, a case-matching study should be conducted in our future research.

In conclusion, elderly people with mild-to-moderate dementia might experience benefits from using the STC6FA in TC training, because they can learn and 


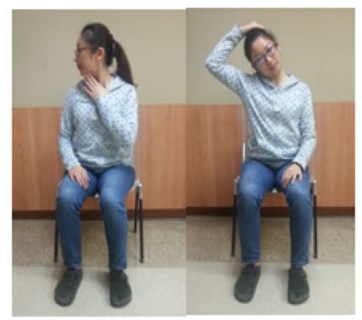

(a) Neck stretch

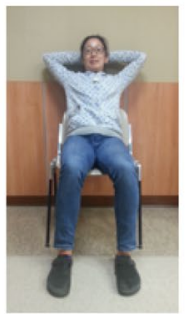

(e) Chest stretch

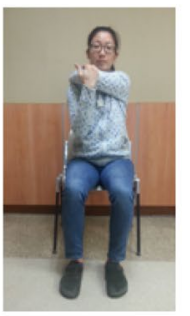

(b) Shoulder stretch

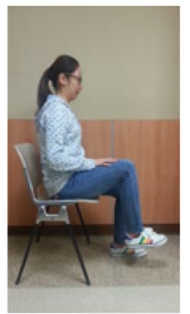

(f) Hip flexion

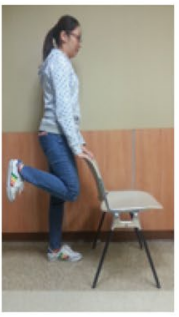

(g) Knee flexion

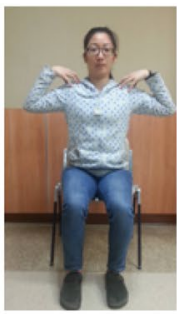

(c) Shoulder circles

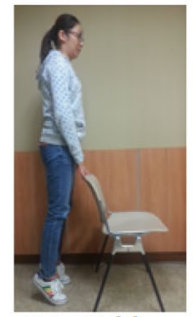

(h) Ankle plantarflexion

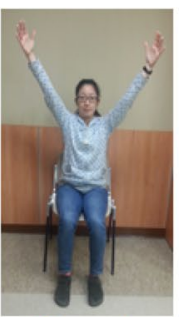

(d) Upper back extension exercise

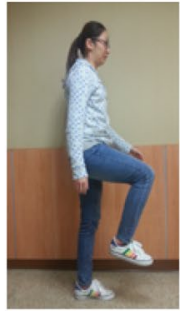

(i) Step exercise

Fig. 7 Stretching exercise of the control group

Fig. 8 Flow diagram of the experiment

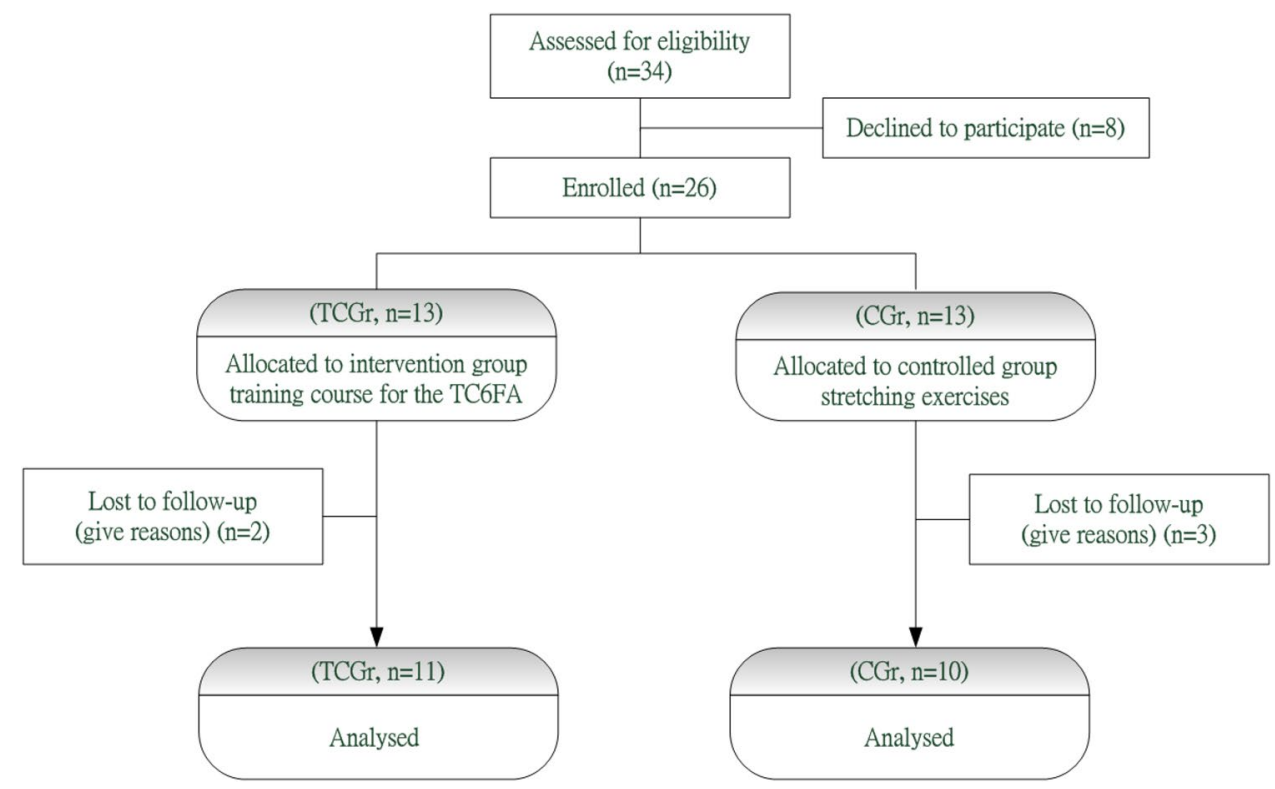

Table 1 Results of demographic data and Clinical Dementia Rating Scale (CDR)

\begin{tabular}{lccl}
\hline & CGr $(\mathrm{n}=10)$ & TCGr $(\mathrm{n}=11)$ & $p$ value \\
\hline Age (years) & $76.8 \pm 6.9$ & $76.7 \pm 11.0$ & 0.986 \\
Body height $(\mathrm{cm})$ & $149.6 \pm 8.4$ & $155.8 \pm 10.2$ & 0.149 \\
Body weight $(\mathrm{kg})$ & $53.9 \pm 6.4$ & $63.3 \pm 9.1$ & $0.013^{*}$ \\
CDR & $0.9 \pm 0.2$ & $0.8 \pm 0.6$ & 0.881 \\
\hline
\end{tabular}

$* p<0.05$ perform TC without depending on their memories of martial arts practices. The STC6FA can significantly improve the balance control of AD patients. Improved balance may reduce the risk of backward falls and fallrelated injuries, which can compromise the mobility of AD patients. Therefore, STC6FA could be a promising exercise modality for supporting elderly people with AD. 
Table 2 Results of balance test and mental health status

\begin{tabular}{|c|c|c|c|c|}
\hline & Pre-test & 8th week & $\begin{array}{l}\text { Within- } \\
\text { group } \\
p \text { value }\end{array}$ & $\begin{array}{l}\text { Between- } \\
\text { groups } \\
p \text { value }\end{array}$ \\
\hline \multicolumn{5}{|l|}{ SOT } \\
\hline \multicolumn{5}{|l|}{ MMSE } \\
\hline TCGr & $17.64 \pm 7.42$ & $19.09 \pm 7.56$ & 0.137 & 0.747 \\
\hline CGr & $21.20 \pm 4.19$ & $22.10 \pm 2.33$ & 0.733 & \\
\hline \multicolumn{5}{|c|}{ Equilibrium score } \\
\hline TCGr & $45.9 \pm 21.7$ & $54.7 \pm 15.9$ & $0.009 *$ & 0.397 \\
\hline CGr & $50.5 \pm 10.3$ & $55.9 \pm 15.0$ & 0.263 & \\
\hline \multicolumn{5}{|l|}{$R W S$} \\
\hline \multicolumn{5}{|c|}{ MVL (deg/sec) } \\
\hline \multicolumn{5}{|l|}{$\mathrm{L} / \mathrm{R}$} \\
\hline TCGr & $4.4 \pm 0.8$ & $5.0 \pm 1.1$ & 0.202 & 0.225 \\
\hline CGr & $5.3 \pm 1.5$ & $5.2 \pm 1.5$ & 0.331 & \\
\hline \multicolumn{5}{|l|}{$\mathrm{F} / \mathrm{B}$} \\
\hline TCGr & $1.7 \pm 0.5$ & $2.6 \pm 0.8$ & $0.015^{*}$ & 0.140 \\
\hline $\mathrm{CGr}$ & $2.2 \pm 0.9$ & $2.4 \pm 0.9$ & 0.443 & \\
\hline \multicolumn{5}{|l|}{ DCL $(\%)$} \\
\hline \multicolumn{5}{|l|}{$\mathrm{L} / \mathrm{R}$} \\
\hline TCGr & $80.8 \pm 5.7$ & $81.5 \pm 3.5$ & 0.858 & 0.705 \\
\hline CGr & $81.9 \pm 2.7$ & $81.0 \pm 5.3$ & 0.812 & \\
\hline \multicolumn{5}{|l|}{$\mathrm{F} / \mathrm{B}$} \\
\hline TCGr & $56.9 \pm 11.1$ & $69.1 \pm 9.8$ & $0.050^{*}$ & 0.165 \\
\hline $\mathrm{CGr}$ & $65.2 \pm 16.9$ & $66.2 \pm 16.9$ & 0.919 & \\
\hline
\end{tabular}

${ }^{*} p<0.05$

MMSE Mini-Mental State Examination, SOT sensory organization test, $R W S$ rhythmic weight-shift, $M V L$ movement velocity, $D C L$ direction control, $L / R$ left and right direction, $F / B$ front and back direction, $T C G r$ TC group, $C G r$ control group

Acknowledgements Sincere appreciation is extended from our group to the Chang Gung Medical Research Program (grant number CMRPG5B0011) for funding this study.

Funding This work was supported by the Chang Gung Medical Research Program (Grant Number CMRPG5B0011).

\section{Compliance with Ethical Standards}

Conflict of interest The authors report no conflicts of interest.

Open Access This article is distributed under the terms of the Creative Commons Attribution 4.0 International License (http://creativecommons.org/licenses/by/4.0/), which permits unrestricted use, distribution, and reproduction in any medium, provided you give appropriate credit to the original author(s) and the source, provide a link to the Creative Commons license, and indicate if changes were made.

\section{References}

1. R. O. C. Department of Household Registration Affairs, MOI. (2018). Report of Taiwan's Ministry of the Interior. Retrieved from https://www.moi.gov.tw/files/site_stuff/321/1/month/ m1-05.xls.

2. Rubenstein, L. Z., \& Josephson, K. R. (2006). Falls and their prevention in elderly people: What does the evidence show? Medical Clinics of North America, 90(5), 807-827. https://doi. org/10.1016/j.mcna.2006.05.013.

3. Rubenstein, L. Z. (2006). Falls in older people: Epidemiology, risk factors and strategies for prevention. Age and Ageing, 35(2), i37-i41. https://doi.org/10.1093/ageing/afl084.

4. Wang, J. Z., Chen, Z., \& Song, Y. (2010). Falls in aged people of the Chinese mainland: Epidemiology, risk factors and clinical strategies. Ageing Research Reviews, 9, S13-S17. https://doi. org/10.1016/j.arr.2010.07.002.

5. Woollacott, M., \& Shumway-Cook, A. (2002). Attention and the control of posture and gait: A review of an emerging area of research. Gait and Posture, 14(1), 1-14. https://doi.org/10.1016/ S0966-6362(01)00156-4.

6. Pettersson, A., Olsson, E., \& Wahlund, L. (2005). Motor function in subjects with mild cognitive impairment and early Alzheimer's disease. Dementia and Geriatric Cognitive Disorders, 19, 299-304. https://doi.org/10.1159/000084555.

7. Persad, C. C., Jones, J. L., Ashton-Miller, J. A., Alexander, N. B., \& Giordani, B. (2008). Executive function and gait in older adults with cognitive impairment. The Journals of Gerontology, Series A: Biological Sciences and Medical Sciences, 63(12), 1350-1355. https://doi.org/10.1093/gerona/63.12.1350.

8. Scherder, E., Eggermont, L., Swaab, D., van Heuvelen, M., Kamsma, Y., de Greef, M.,... Mulder, T. (2007). Gait in ageing and associated dementias; its relationship with cognition. Neuroscience and Biobehavioral Reviews, 31(4), 485-497. https ://doi.org/10.1016/j.neubiorev.2006.11.007.

9. Harlein, J., Dassen, T., Halfens, R. J., \& Heinze, C. (2009). Fall risk factors in older people with dementia or cognitive impairment: A systematic review. Journal of Advanced Nursing, 65(5), 922-933. https://doi.org/10.1111/j.1365-2648.2008.04950.x.

10. Faber, M. J., Bosscher, R. J., Chin A Paw, M. J., \& van Wieringen, P. C. (2006). Effects of exercise programs on falls and mobility in frail and pre-frail older adults: A multicenter randomized controlled trial. Archives of Physical Medicine and Rehabilitation, 87(7), 885-896. https://doi.org/10.1016/j. apmr.2006.04.005.

11. Blondell, S. R., Hammersley-Mather, R., \& Veerman, J. L. (2014). Does physical activity prevent cognitive decline and dementia? A systematic review and meta-analysis of longitudinal studies. BioMed Central Public Health, 14, 510. https://doi. org/10.1186/1471-2458-14-510.

12. Venturelli, M. R., Scarsini, R., \& Schena, F. (2011). Six-month walking program changes cognitive and ADL performance in patients with Alzheimer. American Journal of Alzheimer's Disease and other Dementias, 26(5), 381-388. https://doi. org/10.1177/1533317511418956.

13. Wong, A. M., Chou, S. W., Huang, S. C., Lan, C., Chen, H. C., Hong, W. H,... Pei, Y. C. (2011). Does different exercise have the same effect of health promotion for the elderly? Comparison of training-specific effect of Tai Chi and swimming on motor control. Archives of Gerontology and Geriatrics, 53(2), e133-e137. https ://doi.org/10.1016/j.archger.2010.07.009.

14. Wong, A. M., Pei, Y. C., Lan, C., Huang, S. C., Lin, Y. C., \& Chou, S. W. (2009). Is Tai Chi Chuan effective in improving lower limb response time to prevent backward falls in the elderly? Age, 31(2), 163-170. https://doi.org/10.1007/s11357-009-9094-3. 
15. Pei, Y. C., Chou, S. W., Lin, P. S., Lin, Y. C., Hsu, T. H., \& Wong, A. M. (2008). Eye-hand coordination of elderly people who practice Tai Chi Chuan. Journal of the Formosan Medical Association, 107(2), d103-d110. https://doi.org/10.1016/S0929 $-6646(08) 60123-0$.

16. Lam, L. C., Chau, R., Wong, B. M., Fung, A. W., Lui, V. W., Tam, C. C.,... Chan, W. M. (2011). Interim follow-up of a randomized controlled trial comparing Chinese style mind body (Tai Chi) and stretching exercises on cognitive function in subjects at risk of progressive cognitive decline. International Journal of Geriatric Psychiatry, 26(7), 733-740. https://doi.org/10.1002/gps.2602.

17. Wayne, P. M., Walsh, J. N., Taylor-Piliae, R. E., Wells, R. E., Papp, K. V., Donovan, N. J., et al. (2014). Effect of Tai Chi on cognitive performance in older adults: Systematic review and meta-analysis. Journal of the American Geriatrics Society, 62(1), 25-39. https://doi.org/10.1111/jgs.12611.

18. Wang, F., Lee, E. K., Wu, T., Benson, H., Fricchione, G., Wang, W., et al. (2014). The effects of Tai Chi on depression, anxiety, and psychological well-being: A systematic review and metaanalysis. International Journal of Behavioral Medicine, 21(4), 605-617. https://doi.org/10.1007/s12529-013-9351-9.

19. Perry, R. J., \& Hodges, J. R. (1999). Attention and executive deficits in Alzheimer's disease: A critical review. Brain: A Journal of Neurology, 122(3), 383-404. https://doi.org/10.1093/brain /122.3.383.

20. Lee, H. W., Liang, Y. Z., Chiou, W. K., Lan, H. C., \& Wong, A. M. K. (2010). Satisfaction with Tai Chi 6-form sports apparatus in elderly people and stroke patients. Taiwan Geriatrics and Gerontology, 5(3), 204-215.

21. McKhann, G. M., Knopman, D. S., Chertkow, H., Hyman, B. T., Jack Jr., C. R., Kawas, C. H.,... Phelps, C. H. (2011). The diagnosis of dementia due to Alzheimer's disease: recommendations from the National Institute on Aging-Alzheimer's Association workgroups on diagnostic guidelines for Alzheimer's disease. Alzheimer's \& Dementia: The Journal of the Alzheimer's Association, 7(3), 263-269. https://doi.org/10.1016/j.jalz.2011.03.005.

22. Tang-Wai, D. F., Knopman, D. S., Geda, Y. E., Edland, S. D., Smith, G. E., Ivnik, R. J.,... Petersen, R. C. (2003). Comparison of the short test of mental status and the mini-mental state examination in mild cognitive impairment. Archives of Neurology, 60(12), 1777-1781. https://doi.org/10.1001/archneur.60.12.1777.

23. Prince, M., Bryce, R., Albanese, E., Wimo, A., Ribeiro, W., \& Ferri, C. P. (2013). The global prevalence of dementia: A systematic review and metaanalysis. Alzheimer's \& Dementia: The Journal of the Alzheimer's Association, 9(1), 63-75. https://doi. org/10.1016/j.jalz.2012.11.007.

24. Homann, B., Plaschg, A., Grundner, M., Haubenhofer, A., Griedl, T., Ivanic, G.,... Homann, C. N. (2013). The impact of neurological disorders on the risk for falls in the community dwelling elderly: A case-controlled study. British Medical Journal Open, 3(11), e003367. https://doi.org/10.1136/bmjopen-2013-003367.

25. Davis, J. C., Bryan, S., Marra, C. A., Sharma, D., Chan, A., Beattie, B. L.,... Liu-Ambrose, T. (2013) An economic evaluation of resistance training and aerobic training versus balance and toning exercises in older adults with mild cognitive impairment. PLoS ONE, 8(5), e63031. https://doi.org/10.1371/journal.pone.00630 31.

26. Chang, Y. K., Pan, C. Y., Chen, F. T., Tsai, C. L., \& Huang, C. C. (2012). Effect of resistance-exercise training on cognitive function in healthy older adults: A review. Journal of Aging and Physical Activity, 20(4), 497-517.

27. Sobol, N. A., Hoffmann, K., Frederiksen, K. S., Vogel, A., Vestergaard, K., Brændgaard, H.,... Beyer, N. (2016). Effect of aerobic exercise on physical performance in patients with Alzheimer's disease. Alzheimer's \& Dementia: The Journal of the Alzheimer's Association, 12(12), 1207-1215. https://doi.org/10.1016/j. jalz.2016.05.004.

28. Logghe, I. H., Zeeuwe, P. E., Verhagen, A. P., Wijnen-Sponselee, R. M., Willemsen, S. P., Bierma-Zeinstra, S.,... Koes, B. W. (2009). Lack of effect of Tai Chi Chuan in preventing falls in elderly people living at home: a randomized clinical trial. Journal of the American Geriatrics Society, 57(1), 70-75. https://doi.org /10.1111/j.1532-5415.2008.02064.x.

29. Tsang, W. W. (2013). Tai Chi training is effective in reducing balance impairments and falls in patients with Parkinson's disease. Journal of Physiotherapy, 59(1), 55. https://doi.org/10.1016/ S1836-9553(13)70148-6. 